\title{
Nurses' role in advancing primary health care: a call to action
}

Jeanne Besner Research Initiatives in Nursing and Health, Calgary Health Region, Alberta, Canada

\begin{abstract}
It is no coincidence that in setting the agenda for primary health care (PHC), it was acknowledged that nurses would have to play a major role in helping to shift the health system away from a predominant focus on illness and cure, toward increased attention to health promotion and disease prevention. The goal in primary health care is to 'protect and promote the health of all people of the world' where health is defined in a positive sense as well-being, not merely as absence of illness. Health so defined has always been the concern of nurses.

In this paper, the relationship between modern professional nursing and PHC will be discussed. The notion that advancing the primary health care agenda is inherently within the domain of nursing practice will be developed, citing examples from a PHC project that involved nurses working with family physicians in a primary care practice. The position will be taken that primary health care provides an opportunity to revitalize the nursing workforce, by recapturing the vision of early nursing leaders, such as Florence Nightingale, who recognized the need for social reform in improving population health and understood nurses' role in achieving that.
\end{abstract}

Key words: community nursing; nursing leadership in primary health care; practice nursing

If the millions of nurses in a thousand different places articulate the same ideas and convictions about primary health care, and come together as one force, then they could act as a powerhouse for change. I believe that such a change is coming, and that nurses around the globe, whose work touches each of us intimately, will greatly help to bring it about. (Mahler, 1985)

It has been almost 20 years since nurses were challenged (Mahler, 1985) to take a lead in advancing primary health care throughout the world (WHO/UNICEF, 1978). Yet, many would agree that we have made relatively little global progress in realizing this social goal. Although PHC reflects the values and principles that have always guided registered nurses in their practice,

Address for correspondence: Jeanne Besner, Research Initiatives in Nursing and Health, Calgary Health Region, 10101 South Port Road SW, Calgary, Alberta, Canada, TSW $3 \mathrm{~N} 2$. there is substantial evidence that the call to action toward 'health for all' has not resonated with a majority of nurses, particularly with those employed in acute care settings. If nurses are to play leadership roles in advancing the PHC agenda, they must understand the relationship that exists between PHC and professional nursing practice and refocus their practice as necessary.

In this paper, the relationship between PHC and modern professional nursing is discussed and illustrated. The notion that advancing the primary health care agenda is inherently within the domain of nursing practice is developed, citing examples from a Canadian PHC project that involved registered nurses working with family physicians in a primary care practice. The position is taken that primary health care provides an opportunity to revitalize the nursing workforce, by recapturing the vision of early nursing leaders, such as Florence Nightingale, who recognized the need for health and social reform in improving population health. 


\section{Primary health care}

The driving force behind the global primary health care movement was the recognition that health is a fundamental human right that is inequitably distributed within and between the countries of the world. A PHC focused health system is aimed at improving population health and wellness (i.e., quality of life). This requires that individuals and communities accept greater responsibility for their own health, supported by well coordinated and appropriate multidisciplinary teams of health care providers whose work is closely aligned with that of other social and economic sectors, since 'health cannot be attained by the health sector alone' (WHO/UNICEF, 1978: 40). PHC reform is aimed at building a sustainable, coordinated health system focused on improving population health by ensuring that the right care is provided to the right people, by the most appropriate provider, in the right setting and using the most suitable and cost-effective technology. Technology in this sense refers to the structure and delivery of health services, the deployment of human resources, and medical equipment, pharmaceutical agents or new interventions and techniques (Alberta Association of Registered Nurses, 2003).

The PHC approach highlights the complexity involved in any effort to improve population health and addresses the need for fundamental social change targeted at the broad determinants of health (e.g., income, social status, social supports, etc.). Health promotion is defined as the process of enabling people to increase control over and improve their health (World Health Organization, Health and Welfare Canada, Canadian Public Health Association, 1986). Thus, health promotion is directed at helping individuals, families and/or communities develop the competence and capabilities they require to gain control over day to day life events or circumstances, thereby achieving a sense of wellbeing. Instead of focusing almost exclusively on curative or rehabilitative services, in this 'positive' view of health, practitioners focus attention on the deeper causes of illness, such as poor housing or unemployment, and seek to mobilize personal and community resources in enabling people to 'become agents of their own development' (World Health Organization, 1978).

\section{Nursing, health and primary health care}

It is clear from the writings of Florence Nightingale - the founder of modern professional nursing - that she viewed health as a concept that was to a large extent devoid of the idea of illness. She wrote Notes on Nursing $(1860 / 1969)$ with the intent of 'giving hints for thought to women who have personal charge of others... distinct from medical knowledge, which only a profession can have' (p. xi). In earlier writings, she seemed not to see a need for nurses to possess any medical knowledge, but she eventually altered her position as evidence of the germ theory changed the nature of nursing. Nonetheless, she clearly did not situate nursing primarily within the medical paradigm, for she notes 'experience teaches me that nursing and medicine must never be mixed up' (quoted in Baly, 1991: 68). She made little distinction, however, between nursing knowledge and health knowledge. 'The same laws of health or of nursing, for they are in reality the same, obtain among the well as among the sick (Nightingale, 1860/1969: xii). Thus, whether nursing is practised among individuals who are ill, merely at risk of disease or disability or perfectly healthy, there is something unique that defines the practice and is grounded in a body of knowledge that is distinct from medical knowledge. Nightingale defined nursing practice as 'putting (people) in the best possible conditions for nature to restore or to preserve health', where health was viewed as 'not only to be well, but to be able to use well every power we have' (quoted in Ulrich, 1992: 80). That is similar to the World Health Organization (WHO/UNICEF 1978) concept that primary health care is aimed at enabling people to achieve the highest possible level of health in order that they may contribute to the social and economic development of their communities, which in turn facilitates health development ( $\mathrm{p}$. 39). Nurses often work in settings in which the medical paradigm predominates and frequently perform tasks that support the medical management of patients. That does not mean, however, that nurses operate from within the medical paradigm while performing those tasks. Nursing management of patients incorporates assessment of the physical, psychological, social, cultural and spiritual factors that influence people's ability to 
recover from illness, injury or disability or to achieve optimum health. It is in the implementation of strategies to address the major social and health needs of individuals, families and communities that nursing 'adds value' to the health system.

\section{The nature of nursing}

The principles of PHC are closely aligned with the values of nursing. Nursing, as a profession, is socially mandated to promote strategies for achieving improved population health by developing within its members the skills and knowledge necessary to respond to emerging health needs (American Nurses Association, 1980). The object and philosophical underpinnings of the discipline of nursing are captured in the ANA statement that

Nursing helps to serve society's interests in the area of health.... One of the most distinguishing characteristics of nursing is that it involves practices that are nurtrative, generative, or protective in nature. They are developed to meet the health needs of individuals as integrated persons rather than as biological systems.... Nursing care is provided in an interpersonal process of nurse-with-a-patient, nurse-with-a-family, nurse-with-a-group. (p. 18)

For nurses, the client is always viewed within the context of family (e.g., culture, beliefs and values) and environment (e.g., housing, employment, social support). Since nursing has biological, psychological and social health as an end in view, the outcomes of nursing practice reflect practitioners' contributions to promoting individual and family development during periods of transition or crisis (nurtrative function). There should also be evidence that nursing has influenced changes in health state or behaviour (generative function), in a manner that safeguards respect for individuals and families, and promotes selfreliance (protective function). Nursing assessment is inherently focused on understanding the relationship between determinants of health and actual health status and nursing intervention is directed at helping individuals, families or com- munities develop strategies to achieve improved health and well-being, in the spirit of self-reliance.

In mid-nineteenth century England, the relationship between poverty and ill health could not be denied and a surge of humanitarianism led to the introduction of important health and social reforms. It was in this seedbed of reform that professional nursing first took root. Nightingale felt herself called for a special mission and the provision of trained nurses to help alleviate the suffering of the poor was the fulfilment of that responsibility (Baly, 1991). She was convinced of the importance of preventive social policies and certainly saw that health depended on the whole environment, not just the state of the body. She wrote in 1861, 'It is mere childishness to tell us that it is not important to know what houses people live in. The connection between health and the dwellings of the population is one of the most important that exists' (quoted in Baly, 1991: 39). Nightingale saw concern for humankind and social reform as key features of the work of nurses in her day and that is no different in today's environment.

There are many other examples of early nursing leaders whose entire professional lives were dedicated to the application of nursing science in correcting social inequities between the rich and poor. They were masters in putting health on the agenda of policy makers and were responsible for initiating many social reforms. For example, Lillian Wald (1915) said of the work carried out at the nurses' settlement in East New York 'we do not believe that what we offer is of great consequence unless ... applicable to the problems of the community' (p. 143).

Nursing is directed toward helping to create the environments - physical, social and emotional that are conducive to the attainment of the highest possible level of health and well-being for individuals, families and communities. To quote again from Lillian Wald (1934):

When we went to press for housing reform or for child protection, we always called those most interested - the mothers - into our council, to ask their views on what evils they would most like to see abolished and what they felt the remedy should be.... Now they most admirably formulate and state their convictions as to needed 
changes in law or administration.... During all this time these women ... have had a part in social matters beyond their personal interests and they have felt a growing responsibility to give voice to their opinions on the issues of the day (pp. 18-19).

It would be difficult to find a better description of empowerment or self-care capacity enhancement than is represented in the above passage. It is precisely in their ability to establish clear links between health and social conditions that nurses can make the greatest contribution to health and social reform. The value of nursing to society is, and always has been that entry to health and well-being often occurs through episodes of illness or as a consequence of transitional life events. Through their contact with individuals and families in homes, schools, workplaces and other institutional and community settings, nurses have tremendous opportunities to use knowledge gained at the individual level to effect broad social change.

By giving voice to the knowledge they acquire through their day to day practice, nurses can make a significant contribution to the advancement of primary health care. That does require that nurses assume greater responsibility for focusing on the promotion of health during all interactions with people, whether they are experiencing illness, crisis or a life transition. Effective leadership is nonetheless required to ensure that the potential for harnessing the knowledge, experience, capabilities and commitment of registered nurses in advancing the primary health care agenda becomes reality. For too long, registered nurses in too many settings have seen their scope of practice narrowed to the performance of duties that support the medical management of patients. If nurses are to play their rightful role in helping to shift our health care systems toward primary health care, employers, educators, medical colleagues and nurses themselves will have to value nurses' role in promoting health in all settings where they practice. Nurses will only experience frustration as long as the organizations in which nurses are employed do not support the holistic, family-centred and health-related approach to service delivery that is integral to the discipline of nursing.

\section{Primary health care in action}

In 1998, a primary health care partnership project was established between a group of family physicians and a regional health authority (the region) in a large urban city in western Canada. A public health nurse (full time) and a home care nurse (0.5 FTE) from the region were assigned to the family practice, to collaboratively manage with physicians the health needs of a defined population of approximately 12000 patients. The project was financially supported through a provincial strategy that was aimed at encouraging innovation in the delivery of health services and was facilitated by an alternative funding model that reflected a shift from the traditional fee-forservice payment for physicians in Canada. The primary goals for the project were to optimize the health of individuals served by the practice, eliminate duplication and unnecessary overlaps in service delivery through more appropriate utilization of health providers and create a more integrated system of health care delivery. Key elements of the partnership included a greater focus on population health needs, improving access to health as well as to health care, increased participation of clients in decision-making, and increased attention to opportunities for illness/ injury prevention and health promotion (Aufricht, 2003). The public health nurse played a leadership role in encouraging the application of a population-based approach that integrated prevention and health promotion in planning programmes and services for the practice.

\section{Balancing individual and population health needs}

An important element of the partnership project was to describe the population served by the family physician practice. This afforded an opportunity to better understand how a multidisciplinary team of health professionals could effectively deliver PHC focused services to a group of patients, ensuring provision of the 'right services' by the most appropriate provider. Patient data from the electronic client record were used to create a demographic profile of the practice population by gender and life stage, creating a 'denominator' or context within which 
to better understand the health needs of this group of patients. Analysis of data revealed that the largest single group of patients was adult females (age 20-44), followed by adult males and mature (45-64 years) females.

These data were supplemented with a review of the literature and other information (e.g., annual health status report for the region) to identify major risk factors associated with this population. For example, analysis of regional data indicated that suicide, breast and lung cancer, heart disease, motor vehicle accidents and stroke are among the leading causes of loss of life or productivity in the adult age groups.

Diagnoses related to coughs and colds were the primary reason that women visit a physician, followed by mental health, reproductive and developmental concerns, injuries and hypertension. Coughs and colds are also the leading reason why men visit a physician, followed by joint injuries, neurotic and nonpsychotic disorders, hypertension and otitis media. Regional physician utilization data were consistent with observed patterns for patients registered with the physician practice involved in the project (Shandro, 2001).

Although the physicians in the practice were funded under a capitation model, they continued to perform 'shadow billing' (i.e., as though they were still billing under the feefor-service system) for evaluation purposes. These 'utilization' data were grouped into broad diagnostic categories (e.g., cardiovascular, respiratory) to facilitate analysis of the major underlying reasons for accessing services (i.e., the numerator) from the practice. Analysis of the various types of data revealed significant gaps between what services were being delivered and what could or should be provided for the type of population being served. As a result, new approaches to service delivery were initiated, involving regional personnel and services (e.g., diabetes education clinic) to enhance management of patients with such conditions as diabetes, asthma, depression and cardiovascular risk factors (e.g., hypertension and elevated cholesterol).

One of the most significant impacts of this population-focused approach to addressing the health needs of the practice clients was the increased 'appropriate' use of registered nurses in the practice. Unlike the fee-for-service reimbursement system, the capitation model removed the need for physicians to have direct contact with all patients accessing services in the practice. Registered nurses employed by the family physicians (i.e., practice nurses), as well as the public health and home care nurses were thus able to assume greater responsibility in providing comprehensive services to clients of the practice. This introduced greater flexibility in how services were delivered. By focusing on previously unmet needs in the population (e.g., initiating routine screening for stress and family violence in the adult female population) there was greater opportunity to broaden the range of services offered. The public health nurse was instrumental in enabling the practice nurses to fully utilize the knowledge and skill they possessed in assessing the physical, emotional and social needs of the practice population and in educating clients on health issues. This contributed to enhanced quality of work life for all providers, as well as better service to clients.

Without data obtained through comprehensive analysis of the health needs of the specific population served by the practice, it is unlikely that the public health nurse could have identified for other members of the health team what needed to be done differently to shift the focus of the practice away from 'medical management' of the population toward primary prevention and health promotion. For nurses to use the knowledge they gain through contacts with individuals to effect health and social change, they must be able to record, analyse and then disseminate data about how people's social and environmental contexts influence their health. Current health records are woefully inadequate in that respect. It is essential that nurses, funding bodies and employers demonstrate commitment to ensuring that nursing assessment data (e.g., social, emotional, cultural, spiritual determinants) are incorporated into the development of personal electronic records. In building health information infrastructures, we must not make the mistake of simply converting existing medical records into electronic formats. That will simply perpetuate the inability to link social determinants to health and will continue the relative invisibility of nurses' contributions to population health. 


\section{Access to health as well as to health care}

One of the aims of the partnership project was to provide 24 hour, 7 days per week access to services for patients of the practice population. This was achieved in part through implementation of computer assisted Nurse Telecare services (often conceptualized as telephone triage). Registered nurses employed by the region provided 'afterhours' telecare coverage and practice nurses staffed the telephone line during regular office hours. Nurses thus became the first point of access for patients to the practice, which provided increased opportunities to focus on helping patients take greater responsibility for their own health. Telephone call data were analysed to determine the nature and frequency of calls made to the practice and identify repeat callers whose frequent calls could suggest a need for education or support, to be provided by the public health nurse or another health professional, such as a mental health specialist. Every opportunity was taken to assist patients in managing their symptoms at home when appropriate and to focus on development of self-help strategies that could be used to manage subsequent minor ailments, thus reducing ongoing reliance on physicians and nurses. Telecare nurses considered themselves partners with physicians in the delivery of care.

\section{Appropriate use of technology}

Although telephone advice provided by nurses is gaining popularity in a number of jurisdictions, most such systems operate in parallel with other primary care services. In this project, IT connectivity established between the practice, the region and physicians' homes enhanced providers' ability to deliver seamless care to patients. Nurses had access to the electronic patient record while providing telephone services, as did the on-call physician who could also access client records from home when the nurse sought consultation. Information from all telephone calls was documented on the client record, ensuring informed management of patients and effective multidisciplinary collaboration in care delivery. IT connectivity among team members was identified as a major strength of this project, in that it promoted enhanced communication among members of the team and improved coordination of care. Evaluation of the project confirmed that registered nurses were effective in increasing people's self-care capacities. Furthermore, call screening and patient education by nurses reduced the number of unnecessary patient visits to the practice or walk-in clinics and inappropriate utilization of emergency departments for minor conditions. Patients expressed satisfaction with the quality of and manner in which services were provided. The use of standardized protocols related to the triage system or developed collaboratively by team members helped ensure continuity of care and enabled the team to identify the most appropriate provider needed to address patient concerns in a given situation (Gerlock et al., 2003).

Integration of regional staff into the practice improved collaboration and teamwork among physicians, nurses and other members of the health team (e.g. mental health specialists, endocrinologist and diabetes educators). Collaborative practice and effective teamwork resulted in reduced duplication and fragmentation of services. For example, there was a significant reduction in the number of duplicate well baby and preschool examinations that had formerly been conducted separately by the family physicians and public health nurses. Increased communication between physicians and the home care nurse resulted in better coordination of services and improved quality of care (Gerlock et al., 2003).

At the core of primary health care is the need to transform the health system from one in which a multitude of providers focusing on illness care and working primarily in silos begin to work collaboratively both within and across the health sector to deliver a broad array of health promoting services. This project demonstrated the benefits of infusing primary health care principles in the delivery of primary care services. Unfortunately, it remains difficult for health care organizations to fully support nurses and other providers to work collaboratively in increasing prevention and promotion strategies, as long as the concentration of government health resources remaines fixed on curative services and institutions. 


\section{Disease/injury prevention and health promotion}

There is no area of practice in which the role of nurses is more clearly complementary to that of physicians than in disease prevention and health promotion. In this project, registered nurses played a key role in leading and facilitating initiatives that were aimed at refocusing service delivery toward primary prevention. Analysis of demographic data and risk factors associated with the practice population identified a need to target women's health issues and to concentrate more attention on reduction of cardiovascular risk for the adult population. The public health nurse assumed a leadership role in involving physicians, clients, practice nurses and other office and regional personnel in the development of care pathways to ensure that all clients visiting the practice would be assessed for the presence of risk factors. For example, a women's health initiative was implemented to develop programmes and policies that were more responsive to women's health needs. Women visiting the practice for routine check-ups or episodic health issues are now asked about their emotional well-being (e.g., unusual stress, physical/mental abuse) and receive lifestyle assessment (exercise history, tobacco use, alcohol/substance abuse, seat belt use, etc.) in addition to screening for cancer and heart disease, as appropriate. Using this systematic, focused approach to identifying population health needs led to development of targeted health education programmes and increased preventive screening (Gerlock et al., 2003). Patient involvement on a patient advisory committee established for this project provided some assurance of the acceptability of programme changes that evolved over the course of the project. Furthermore, clients who participated on committees became informed 'consumers' whose knowledge of health care issues increased dramatically over time. They became outspoken advocates of the multidisciplinary care model that incorporates an expanded role for nurses and involvement of a broader group of professionals located at, or associated with the family practice.

\section{Conclusion}

The promotion of health has long been considered the purview of nursing. Within the current health reform environment, nurses have been challenged to reorient their practice to be consistent with primary health care philosophy and principles (Clarke, 1995). The primary health care approach demands that more effort be made by nurses to empower clients to assume control over personal, social and political determinants of health. Nursing has a key role to play in developing new approaches to improving population health - approaches that recognize the essential role of clients as active participants and that emphasize the positive view of health. If nurses are to respond to the challenge of demonstrating that their practice makes a viable difference to the health of individuals and populations, they will need to be guided by appropriate conceptual models that align their practice with that purpose. Management expectations will also need to be revised, since practice is to a large extent directly influenced by organizational and programme structures (Besner, 1999). At the very least, the nursing skills required to engage individuals and families meaningfully in goal setting and development of health promoting strategies will need to be as highly valued and nurtured by employers as is clinical expertise. Primary health care is an approach that offers the opportunity of using the education, knowledge and skills of registered nurses for the greatest benefit of patients and the health system. Unless nurses are able to make explicit their unique focus on the health of individuals, they risk missing the opportunity offered through health care reform to reaffirm their role in assisting individuals, families and communities to function at the highest level of their potential (International Council of Nurses, 1996). If nurses' health promoting role is ever to be fully realized, changes will need to occur at the academic, practice and policy levels (International Council of Nurses, 1996; Mahler, 1985).

\section{References}

Alberta Association of Registered Nurses. 2003: Primary health care. Edmonton, AB: Alberta Association of Registered Nurses.

Aufricht, P. 2003: Crowfoot village family practice primary health care reform pilot project. Final Report 30 April 2003. Unpublished manuscript. Calgary, AB, Canada: Author.

American Nurses Association. 1980: Nursing. A social policy statement. Kansas City, MO: American Nurse Association. 
Baly, M. 1991: As Miss Nightingale said...Florence Nightingale through her sayings. A Victorian perspective London: Scutari Press.

Besner, J. 1999: A theory-based approach to establishing effectiveness in a public health nursing program. Unpublished doctoral thesis.

Clarke, H.F. 1995: Research-based practice in community health nursing. In Stewart, M.J. editor, Community nursing: promoting Canadians' health. Toronto: W.B. Saunders Canada, 577-99.

Gerlock, A., Woodhead-Lyons, S. and Church, J. 2003: Primary care partnerships - the Crowfoot project. Final Evaluation Report. Unpublished manuscript.

International Council of Nurses. 1996: The value of nursing in a changing world. Geneva:International Council of Nurses.

Mahler, H. 1985: Nurses lead the way. New Zealand Nursing Journal October, 10-11.
Nightingale, F. 1969: Notes on nursing. New York: Dover (Original work published in 1860).

Shandro, G. 2001: Primary care partnerships project (CVFP/CHR partnership - Health Innovation Fund project). Assessment Report. Unpublished manuscript. Calgary, AB: Author.

Ulrich, B. T. 1992: Leadership and management according to Florence Nightingale. Norwalk, CT: Appleton \& Lange.

Wald, L. D. 1915: The House on Henry Street. New York: Henry Holt.

- 1934: Windows on Henry Street. Boston: Little Brown.

WHO/UNICEF. 1978: Primary health care. Report of the International Conference on Primary Health Care Alma- Ata, USSR, 6-12 September 1978. Jointly sponsored by the World Health Organization and the United Nations Children's Fund. Geneva: World Health Organization. 